\title{
Vitamin D levels and vitamin D receptor genetic variants in Egyptian cardiovascular disease patients with and without diabetes
}

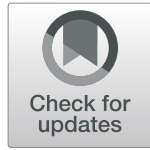

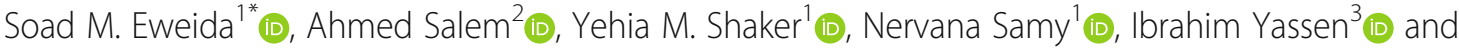 \\ Rania Hassan Mohamed² (iD
}

\begin{abstract}
Background: 25-Hydroxyvitamin D (Vit.D) levels associated with cardiovascular disease (CVD) may vary according to genetic variants in the vitamin D receptor (VDR) gene. However, the existing results are not conclusive in the Egyptian population, where diabetes mellitus is a common CVD risk factor. The purpose of the study was to evaluate the role of VDR polymorphism in Egyptian patients with CVD by studying the association of the rs2228570 (Fokl) and rs1544410 (Bsml) single nucleotide polymorphisms (SNPs) of the VDR gene and serum levels of Vit.D with several CVD risk factors in patients with and without diabetes mellitus. We studied the genotypes for rs 2228570 (Fokl) and rs1544410 (Bsml) SNPs of the VDR gene in 382 Egyptian patients (120 CVD patients with diabetes, 124 CVD patients without diabetes, 69 diabetic patients without CVD and 69 healthy individuals). We also determined the serum levels of Vit.D, insulin, lipids, fasting blood glucose (FBG), and the body mass index (BMI).
\end{abstract}

Results: The distribution of genotypes and allelic frequencies of the rs2228570 (Fokl) and rs1544410 (Bsml) SNPs of the VDR gene was significant in CVD patients $(p<0.001)$. The level of Vit.D was significantly lower in patients with CVD and diabetes compared to those without diabetes $(p<0.001)$. Moreover, there was a significant association between Vit.D level and the selected SNPs with serum lipids, BMI, FBG, and insulin levels in CVD patients with or without diabetes.

Conclusion: The level of Vit.D and the distribution of VDR polymorphisms are associated with risk of CVD in Egyptian patients with or without diabetes. These results suggest that VDR polymorphisms may be potential diagnostic biomarkers for CVD susceptibility.

Keywords: Cardiovascular disease, Diabetes, Vitamin D, VDR gene polymorphism

\section{Background}

Cardiovascular disease (CVD) is a major health problem and a common cause of morbidity and mortality in both high and low-income countries $[1,2]$. According to the World Health Organization (WHO), CVDs account for 17.9 million deaths worldwide and this number is expected to increase to 23.6 million by 2030. In Egypt, CVDs account for $40 \%$ of total deaths [3, 4]. Many

\footnotetext{
*Correspondence: soad.nrc@gmail.com

'Department of Biochemistry, National Research Centre, Dokki, Giza, Egypt

Full list of author information is available at the end of the article
}

factors, such as type 2 diabetes (T2D), hypercholesterolemia, obesity, physical inactivity, and smoking, have been found to have significant effects on CVD risk, progression, and severity through affecting carbohydrate and lipid metabolism, blood pressure, vascular function, and atherogenesis [5]. The incidence of CVD is 2-8 folds higher in T2D patients than those without T2D, as hyperglycemia may impair both arterial and endothelial functions leading to vascular inflammation, foam cell formation, thrombosis, and increased arterial stiffness [6]. Common cardiovascular disorders result from a combination of metabolic factors and genetic alterations. 
Recent genomic studies suggest that the use of susceptibility variants may be important for the diagnosis and treatment of CVD [7].

Vitamin D (Vit.D) has attracted attention as it is linked to several biological processes such as bone mineral homeostasis, management of cell proliferation, and modulation of immune responses [8]. Vit.D is obtained from diet and after exposure to the sun. The inactive form of Vit.D is converted in the liver to $25-(\mathrm{OH})-\mathrm{D}$ followed by $1 \alpha$-hydroxylation in the kidney yielding the active Vit.D metabolite $1,25-(\mathrm{OH})_{2}-\mathrm{D}[9,10]$. Vit.D deficiency has been linked to CVD and T2D, as it regulates endothelial, smooth and cardiac muscle cell functions, blood pressure; in addition, it maintains both glucose tolerance and insulin release [11]. Several epidemiological studies have reported the association between Vit.D deficiency and CVD and its risk factors, such as obesity, T2D, insulin resistance, dyslipidemia and hypertension [12-15].

The majority of Vit.D actions are mediated by vitamin D receptor (VDR). The VDR gene is mapped on chromosome 12q12-q14 consisting of eight proteincoding exons and six untranslated exons. Several single nucleotide polymorphisms (SNPs) have been expressed in the VDR gene that could potentially alter its expression and activation. The most studied SNPs of the VDR gene are rs2228570 (FokI) in exon 2, rs1544410 (BsmI) and rs7975232 (ApaI) in intron 8, and rs731236 (TaqI) in exon 9 [16]. VDR polymorphism has been reported to be associated with Vit.D level and function. VDR polymorphism showed significant association with lower serum levels of Vit.D in obese Egyptian women [14], and might lead to Vit.D deficiency due to VDR dysfunction in patients with vitiligo [17]. Besides its effect on Vit.D status [12], some VDR SNPs have been associated with several diseases such as T2D [18], Parkinson's disease [10], and CVD [19] in several populations. Furthermore, VDR variants have been related not only to CVD but also to different CVD risk factors. The ff genotype at FokI SNP of VDR is associated with dyslipidemia in China, while the FF genotype predicts higher HDL-cholesterol levels [20]. However, some results are inconsistent. Nakhl et al. [21] reported an association between VDR FokI polymorphism and CVD in the Mediterranean region, while Panet et al. [22] reported no association of both FokI and BsmI polymorphisms with CVD in the Chinese population. However, in relation to CVD risk factors in the Egyptian population, few VDR SNPs have been analyzed [23] and whether VDR affects Vit.D levels or could be a risk factor for CVD is still not well elucidated in Egyptian patients. This study aims to correlate the Vit.D levels and frequency of the VDR gene polymorphisms (rs2228570 FokI and rs1544410 BsmI) in
Egyptian patients with cardiovascular disease with and without diabetes, to the clinical characteristics and laboratory findings at diagnosis of CVD.

\section{Methods \\ Study design and subjects}

A total of 382 subjects from Sayed Galal University Hospital, Cairo, Egypt, were recruited over a period of 5 months, from January to May 2017. All subjects were interviewed and examined by a consultant cardiologist, answered a questionnaire about their age, marital status, job, residence, past medical history (hypertension, diabetes, or any psychiatric disorder), smoking habit and nutrition status, and underwent the following: (1) Full clinical chest and heart examination with special stress on the nature of chest pain (onset, course, duration, site, radiation) and dyspnea (whether at rest or on exertion), history of recent vomiting and hypotensive episodes; (2) 12-lead resting electrocardiogram (ECG), echocardiography and coronary angiography; and (3) measurements of weight, height, diastolic blood pressure (DBP), and systolic blood pressure (SBP). In our study, we have chosen confirmed coronary artery disease (CAD) patients who met the WHO criteria [24] and were verified by whether they had experienced prior episodes of STelevation myocardial infarction or by coronary angiography to represent CVD, because CAD patients were relatively prevalent among cases attending Sayed Galal University Hospital. T2D was diagnosed according to the American Diabetes Association criteria [25]. Subjects were divided into four groups: group A, CVD patients with T2D $(n=120)$; group $B, C V D$ patients without T2D $(n=124)$; group $C$, diabetic patients without CVD $(n=69)$; and group $\mathrm{D}$, healthy controls $(\mathrm{n}=69)$.

\section{Inclusion criteria}

Patients with single or multi-vessel CAD with more than $50 \%$ luminal stenosis on angiography.

\section{Exclusion criteria}

(1) History of type 1 diabetes, coronary surgical intervention, organ transplantation, or renal hemodialysis; (2) pregnancy or any medical condition prone to alter parathyroid hormone (PTH) concentration; (3) valvular or congenital heart diseases, hepatic diseases, chronic lung diseases, cancer, or malabsorption syndrome; (4) patients on medications such as anticonvulsants, systemic glucocorticoids, antidepressants, antipsychotics drugs, supplementation with vitamin $\mathrm{D}$, multivitamin supplements, or bisphosphonates.

The treatment protocol for CAD patients included statin, aspirin, and renin-angiotensin-aldosterone system (RAAS) blockers, whereas diabetic patients were treated with glucose-lowering drugs. All subjects were married 
and resided in Cairo. Males were manual workers, while the females were housewives and all were from low socioeconomic class with no regular exercise in their lifestyle and did not complain of any mood disturbance or any behavioral change in the 2 weeks prior to the study.

\section{Methods}

\section{Sampling}

Approximately $5 \mathrm{~mL}$ of peripheral venous blood were withdrawn after a 12-h fast from all subjects. Serum was separated with blood centrifugation at $3000 \mathrm{~g}$ for $10 \mathrm{~min}$ and stored at $-80{ }^{\circ} \mathrm{C}$ for biochemical analysis.

\section{Biochemical analysis}

Fasting blood glucose (FBG) levels were determined using a kit of linear chemicals (Barcelona-Spain). Serum levels of total cholesterol (TC), triglyceride (TG), and HDL-C were calorimetrically measured using a kit from Stanbio laboratory (USA). LDL-C was calculated via Friedewald's formula as TC-HDL-c-TG/5 [26]. Body mass index (BMI) was calculated as weight $(\mathrm{kg}) /$ height $\left(\mathrm{m}^{2}\right)$. Serum human insulin and Vit.D levels were determined by enzymelinked immunosorbent assays (ELISA) using kits from Immunospec Corporation (USA) and Orgentec Diagnostika GmbH (Germany), respectively, according to the manufacturers' instructions. Homeostatic Model Assessment of Insulin Resistance (HOMA-IR) was calculated as FBG (mg/dL) X insulin (U/L)/405 [27].

\section{Genotyping}

Genomic DNA was extracted from 200 microliters of whole blood samples using QIAamp mini kit (Qiagen, Germany) according to the manufacturer's instructions. $($ rs2228570 C > T FokI) and (rs1544410 A > G BsmI) gene polymorphisms were identified by polymerase chain reaction restriction fragment length polymorphism (PCR-RFLP) assay by DNA amplification with specific primers. Primers were adopted from the study of Chen et al. [28], and were checked for specificity using Basic Local Alignment Search Tool (BLAST; Primer BLAST).

rs2228570 (FokI): Forward: 5' GCA CTG ACT CTG GCT CTG AC 3'

Reverse: $5^{\prime}$ ACC CTC CTG CTC CTG TGG CT3'

rs1544410 (BsmI): Forward: 5' GGAGAC ACA GAT AAG GAA ATA C3'

Reverse: 5' CCG CAA GAA ACC TCA AAT AAC A 3'

In each PCR reaction tube, $12.5 \mu \mathrm{L}$ master mix (Taq polymerase and deoxynucleotide triphosphate mixture), $3 \mu \mathrm{L}$ genomic DNA, $1 \mu \mathrm{L}$ forward primer, $1 \mu \mathrm{L}$ reverse primer and $7.5 \mu \mathrm{L}$ distilled water (DW) were mixed. The PCR cycle was as follows: initial denaturation at $95{ }^{\circ} \mathrm{C}(5$ min), 40 cycles of denaturation at $95{ }^{\circ} \mathrm{C}(15 \mathrm{~s})$, annealing at $72{ }^{\circ} \mathrm{C}(\mathrm{FokI}), 60{ }^{\circ} \mathrm{C}(\mathrm{BsmI})(60 \mathrm{~s})$, and extension at 72 ${ }^{\circ} \mathrm{C}$ (45 s). The amplified PCR products of rs 2228570
(341 bp) and rs1544410 (248 bp) were digested with FokI and $B s m I$ restriction enzymes. Post-digestion, the products were run on a $2 \%$ agarose gel then visualized by a UV transilluminator (Supplementary figure 1).

\section{Statistical analysis}

Statistical analyses were conducted using the Statistical Package for the Social Sciences (SPSS, version 20, Chicago, IL). The Shapiro-Wilk test was used for normality testing. Categorical variables were expressed as numbers (percentages) and compared using the chi-square $\left(x^{2}\right)$ test. Continuous variables were expressed as median (interquartile range, IQR: 25th-75th quartile) and compared using the non-parametric Kruskal-Wallis test. The correlations of vitamin D with other study parameters were assessed using Spearman's rank correlation coefficient. Logistic regression analysis was used to estimate the CVD risk factor. The differences in the frequencies of VDR genotypes between cases and controls were determined by the chi-square $(x 2)$ test. Odds ratio (OR) with 95\% confidence interval (CI) was used for risk estimation. The Hardy-Weinberg equilibrium was tested for every polymorphism by comparing expected and observed genotype frequencies using $\left(\mathrm{X}^{2}\right)$ test. The power analysis was carried out using the PASS software [29]. All statistical analyses were two-sided and a $\mathrm{P} \leq 0.05$ was considered to be statistically significant [30].

\section{Results}

The results revealed that BMI was significantly higher in CVD patients with and without T2D compared to diabetic patients and controls. FBG, insulin, HOMA-IR, TC, TG, LDL-C, SBP, and DBP levels showed a significant increase in CVD patients with T2D when compared to CVD patients without T2D, diabetic patients, and controls. In contrast, HDL-C levels were significantly decreased in both CVD patients with and without T2D compared to controls. The mean Vit.D was significantly lower among CVD with T2D than CVD patients without T2D, diabetic patients, and controls (Fig. 1). Table 1 shows demographic, clinical, and biochemical parameters of all studied groups. The study revealed a significant correlation between Vit.D levels and BMI, insulin, HOMA-IR, TC, TG, LDL-C and HDL-C levels in diabetics, and patients with CVD with and without diabetes (Table 2). Moreover, logistic regression analysis was done to address the predictive independent variables. The results showed that increasing age, BMI and SBP, as well as decreasing FBG, insulin, HOMA-IR, and Vit.D were statistically significantly associated with the presence of CVD (Table 3).

According to VDR gene polymorphism, there was a significant difference in genotypes distribution of rs2228570 FokI and rs1544410 BsmI in all studied 


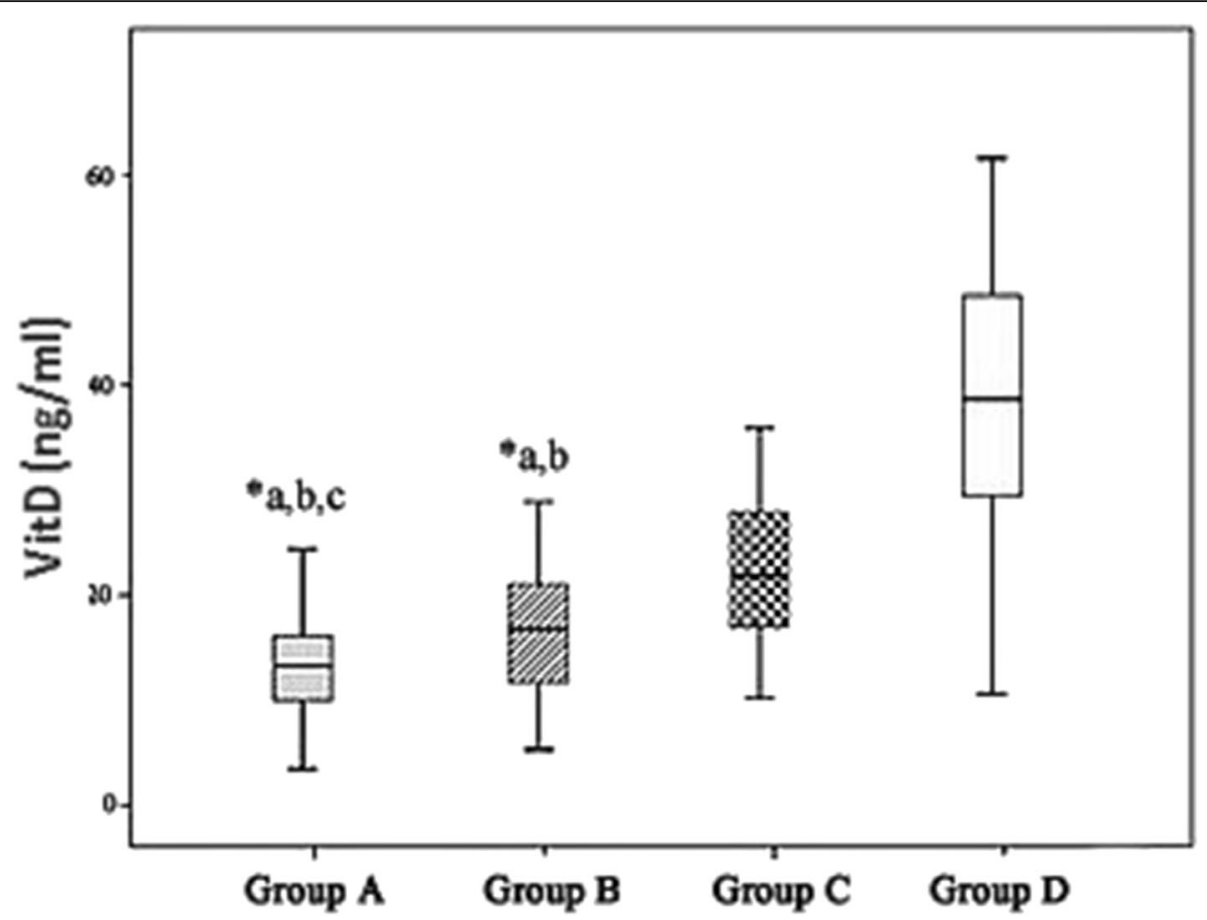

Fig. 1 Box plots of serum Vit. D level in different studied groups. Group A, CVD with T2D; group B, CVD without T2D; group C, diabetic group; group $D$, healthy group; a, significant difference from group $D ; b$, significant difference from group $C ;$, significant difference from group $B$

groups. In addition, the genotype frequencies of both SNPs in all studied groups were in the Hardy-Weinberg equilibrium ( $\mathrm{p}>0.05$ ) (Figs. 2, 3 and Tables 4, 5). Regarding (rs2228570 FokI), there was a significant difference in genotype distribution between CVD without T2D and control group $(\mathrm{p}<0.001)$. Also, a significant association was found between CVD patients with T2D and diabetic patients in both the genotype and allele distributions $(\mathrm{p}<0.01)$, whereas the genotype distribution of the VDR rs2228570 (FokI) showed no significant difference between CVD patients with and without T2D groups. The $\mathrm{T}$ allele was frequently higher in CVD with

Table 1 Demographic, clinical and biochemical parameters

\begin{tabular}{|c|c|c|c|c|c|}
\hline $\begin{array}{l}\text { Groups } \\
\text { Variables }\end{array}$ & $\begin{array}{l}\text { Group A } \\
(n=120)\end{array}$ & $\begin{array}{l}\text { Group B } \\
(n=124)\end{array}$ & $\begin{array}{l}\text { Group C } \\
(n=69)\end{array}$ & $\begin{array}{l}\text { Group D } \\
(n=69)\end{array}$ & $P$ value \\
\hline Age (years) & $58(54.3-63)$ & $57.5(50-62)$ & $54(47-59)$ & $54(50-58)$ & $<0.001$ \\
\hline Sex $(M / F)$ & $52(43.3 \%) / 68(56.7 \%)$ & $94(75.8 \%) / 30(24.2 \%)$ & $27(39.1 \%) / 42(60.9 \%)$ & $36(52.2 \%) / 33(47.8 \%)$ & $<0.001$ \\
\hline BMI $\left(\mathrm{kg} / \mathrm{m}^{2}\right)$ & $29.4(27.7-33)^{a, b}$ & $30.3(28.3-33.2)^{a, b}$ & $26.5(25.3-27.9)$ & $25.9(24.5-27.3)$ & $<0.001$ \\
\hline $\mathrm{SBP}(\mathrm{mmHg})$ & $140(130-150)^{a, b}$ & $130(130-149)$ & 130(120-140) & $130(130-140)$ & 0.005 \\
\hline $\mathrm{DSP}(\mathrm{mmHg})$ & $90(80-90)^{a, b}$ & $80(80-90)$ & $80(77-90)$ & $80(80-90)$ & $<0.001$ \\
\hline FBG (mg/dl) & $189.1(157.8-232.6)^{a, b, c}$ & $89.5(83.8-97.7)^{b}$ & $151.3(137.5-178.1)^{a}$ & $87.5(84.5-94.8)$ & $<0.001$ \\
\hline Insulin ( /I) & $27.1(16.7-40.5)^{a, b, c}$ & $13.4(9.3-22.7)^{a, b}$ & $21.8(18.8-25.5)^{\mathrm{a}}$ & $9.5(7.45-13.5)$ & $<0.001$ \\
\hline HOMA-IR & $11.9(7.1-24.6)^{\mathrm{a}, \mathrm{b}, \mathrm{c}}$ & $2.7(2.0-5.2)^{a, b}$ & $8.9(7.8-11.5)$ & $2.2(1.5-2.9)$ & $<0.001$ \\
\hline $\mathrm{TC}(\mathrm{mg} / \mathrm{dl})$ & $201.2(191.0-220.5)^{a, b}$ & $203.0(185.6-221.9)^{a, b}$ & 175.9 (163.6-193.7) & $176.6(164.7-186.4)$ & $<0.001$ \\
\hline $\mathrm{TG}(\mathrm{mg} / \mathrm{dL})$ & $148.3(128.3-173.1)^{\mathrm{a}, \mathrm{b}, \mathrm{c}}$ & $141.5(117.0-162.2)^{a, b}$ & $95.7(68.1-109.3)^{a}$ & $80.4(66.8-97.5)$ & $<0.001$ \\
\hline $\mathrm{HDL}-\mathrm{C}(\mathrm{mg} / \mathrm{dl})$ & $37.8(28.6-43.6)^{a, b, c}$ & $43.3(34.4-51.5)^{a, b}$ & $50.2(44.8-59.6)^{a}$ & $63.8(54.6-68.7)$ & $<0.001$ \\
\hline LDL-C (mg/dL) & $136.8(124.6-162.8)^{\mathrm{a}, \mathrm{b}, \mathrm{c}}$ & $126.7(114.2-147.4)^{a, b}$ & $111.5(89.7-118.5)^{a}$ & $94.5(88.1-108.7)$ & $<0.001$ \\
\hline Vit.D (ng/ml) & $13.3(10-16.1)^{a, b, c}$ & $17.4(12.4-21.7)^{\mathrm{a}, \mathrm{b}}$ & $23.5(18.7-29.6)^{a}$ & $38.7(29.5-48.5)$ & $<0.001$ \\
\hline
\end{tabular}

Group A, CVD with T2D; group B, CVD without T2D; group C, diabetic group; group D, healthy group; a, significant difference from group D; b, significant difference from group $C ; C$, significant difference from group B. Categorical variables were expressed as numbers (percentages) and compared using chi-square (X2) test. Continuous variables were expressed as median (interquartile range, IQR: 25th-75th quartile) and compared using the non-parametric Kruskal-Wallis test 
Table 2 Associations of Vit.D levels with other clinical and biochemical parameters

\begin{tabular}{|c|c|c|c|c|c|c|c|c|}
\hline \multirow[t]{2}{*}{ Parameter } & \multicolumn{2}{|l|}{$\begin{array}{l}\text { Group A } \\
(n=120)\end{array}$} & \multicolumn{2}{|l|}{$\begin{array}{l}\text { Group B } \\
(n=124)\end{array}$} & \multicolumn{2}{|l|}{$\begin{array}{l}\text { Group C } \\
(n=69)\end{array}$} & \multicolumn{2}{|l|}{$\begin{array}{l}\text { Group D } \\
(n=69)\end{array}$} \\
\hline & $r$ & $P$ value & $r$ & $P$ value & $r$ & $P$ value & $r$ & $P$ value \\
\hline BMI $\left(\mathrm{kg} / \mathrm{m}^{2}\right)$ & $-0.536^{* *}$ & $<0.001$ & $-0.561^{* *}$ & $<0.001$ & $-0.364^{* *}$ & ${ }^{\circ} 0.01$ & -0.068 & 0.578 \\
\hline $\mathrm{SBP}(\mathrm{mmHg})$ & -0.046 & 0.615 & -0.129 & 0.154 & $-0.331^{* *}$ & 0.006 & -0.23 & 0.054 \\
\hline $\mathrm{DSP}(\mathrm{mmHg})$ & -0.094 & 0.309 & -0.133 & 0.139 & -0.105 & 0.389 & -0.154 & 0.206 \\
\hline FBG (mg/dl) & $-0.618^{* *}$ & $<0.001$ & $-0.495^{* *}$ & $<0.001$ & $-0.705^{* *}$ & $<0.001$ & -0.198 & 0.103 \\
\hline Insulin ( /I) & $-0.473^{* *}$ & $<0.001$ & $-0.434^{* *}$ & $<0.001$ & -0.031 & 0.803 & $-0.583^{* *}$ & $<0.001$ \\
\hline HOMA-IR & $-0.584^{* *}$ & $<0.001$ & $-0.477^{* *}$ & $<0.001$ & $-0.345^{* *}$ & ${ }^{<} 0.01$ & -0.014 & 0.910 \\
\hline $\mathrm{TC}(\mathrm{mg} / \mathrm{dl})$ & $-0.684^{* *}$ & $<0.001$ & $-0.675^{* *}$ & $<0.001$ & $-0.779^{* *}$ & $<0.001$ & $-0.562^{* *}$ & $<0.001$ \\
\hline TG (mg/dl) & $-0.505^{* *}$ & $<0.001$ & $-0.664^{* *}$ & $<0.001$ & $-0.402^{* *}$ & ${ }^{<} 0.01$ & $-0.662^{* *}$ & $<0.001$ \\
\hline $\mathrm{HDL}-\mathrm{C}(\mathrm{mg} / \mathrm{dL})$ & $0.713^{* *}$ & $<0.001$ & $0.391^{* *}$ & $<0.001$ & $0.537^{* *}$ & $<0.001$ & $0.756^{* *}$ & $<0.001$ \\
\hline LDL-C (mg/dL) & $-0.772^{* *}$ & $<0.001$ & $-0.524^{* *}$ & $<0.001$ & $-0.810^{* *}$ & $<0.001$ & $-0.645^{* *}$ & $<0.001$ \\
\hline
\end{tabular}

Spearman rank correlation coefficient $(r)$

${ }^{* *}$ Correlation is significant at the 0.01 level

${ }^{*}$ Correlation is significant at the 0.05 level

the T2D group than in CVD without the T2D group with no significant difference between them. The TT genotype was significantly more frequent in CVD with T2D groups than in the diabetic group (Table 4, Fig. 2, Supplementary figure 2). Furthermore, the power analysis was $99 \%$ to detect an effect size of 0.2722 using 6 degrees of freedom chi-square test with a significance level $(\alpha)$ of 0.05 for $(F o k I)$ genotypes in all studied groups. Regarding VDR rs1544410 (BsmI) polymorphism, the results showed a significant difference between CVD with and without T2D as compared to diabetic and control groups, respectively, in genotype and allele distribution. The GG genotype was significantly more frequent in CVD with the T2D group as compared to the diabetic group ( $\mathrm{p}^{<} 0.001$ ). There was a significant difference in $G$ allele distribution between CVD with T2D and CVD without T2D groups ( $\mathrm{p}{ }^{\circ} 0.05$ ) with a risk of having $G$ allele 2 times more in CVD with T2D than CVD without the T2D group (Table 5, Fig. 3, Supplementary figure 3 ). Furthermore, the power analysis was $100 \%$ to detect an effect size of 0.5369 using 6 degrees of freedom chi-square test with a significance level $(\alpha)$ of 0.05 for (BsmI) genotypes in all studied groups.

The distribution of some clinical and biochemical parameters according to the VDR rs2228570 (Fokl) genotype observed in CVD with and without T2D patients is shown in Table 6. Concerning CVD patients without $\mathrm{T} 2 \mathrm{D}$, the results revealed that higher systolic blood

Table 3 Logistic regression analysis for risk factors and biochemical parameters in CVD patients

\begin{tabular}{|c|c|c|c|c|c|c|}
\hline \multirow[t]{2}{*}{ Step $1^{a}$} & \multirow[t]{2}{*}{$\begin{array}{l}\text { Coefficient } \pm \\
\text { S.E. }\end{array}$} & \multicolumn{2}{|c|}{$\begin{array}{l}95 \% \text { Wald } \\
\text { confidence interval }\end{array}$} & \multirow[t]{2}{*}{ Wald $\mathrm{X}^{2}$} & \multirow[t]{2}{*}{ df } & \multirow[t]{2}{*}{$\begin{array}{l}P \\
\text { value }\end{array}$} \\
\hline & & Lower & Upper & & & \\
\hline Age (years) & $0.200 \pm 0.046$ & 1.116 & 1.337 & 18.791 & 1 & ${ }^{<} 0.001$ \\
\hline Sex & $0.094 \pm 0.686$ & 0.286 & 4.217 & 0.019 & 1 & 0.892 \\
\hline BMI $\left(\mathrm{kg} / \mathrm{m}^{2}\right)$ & $0.182 \pm 0.079$ & 1.029 & 1.400 & 5.382 & 1 & ${ }^{<} 0.05$ \\
\hline SBP $(\mathrm{mmHg})$ & $1.639 \pm 0.718$ & 1.261 & 21.043 & 5.210 & 1 & ${ }^{<} 0.05$ \\
\hline $\mathrm{DSP}(\mathrm{mmHg})$ & $-0.050 \pm 0.034$ & 0.889 & 1.017 & 2.141 & 1 & 0.143 \\
\hline FBG (mg/dl) & $-0.048 \pm 0.013$ & 0.928 & 0.979 & 12.725 & 1 & $<^{<} 0.001$ \\
\hline Insulin (u/l) & $-0.202 \pm 0.076$ & 0.703 & 0.949 & 7.014 & 1 & ${ }^{<} 0.01$ \\
\hline HOMA-IR & $0.450 \pm 0.183$ & 1.096 & 2.243 & 6.053 & 1 & ${ }^{<} 0.05$ \\
\hline $\mathrm{TC}(\mathrm{mg} / \mathrm{dl})$ & $0.474 \pm 619.14$ & 0.000 & 0 & 0.000 & 1 & 0.999 \\
\hline TG (mg/dL) & $-0.057 \pm 123.83$ & 0.000 & 0 & 0.000 & 1 & 1.000 \\
\hline HDL-C (mg/dl) & $-0.530 \pm 619.14$ & 0.000 & 0 & 0.000 & 1 & 0.999 \\
\hline LDL-C (mg/dL) & $-0.478 \pm 619.14$ & 0.000 & 0 & 0.000 & 1 & 0.999 \\
\hline Vit.D (ng/ml) & $-0.357 \pm 0.078$ & 0.601 & 0.815 & 21.135 & 1 & $<0.001$ \\
\hline
\end{tabular}

Variables are entered on step 1, $d f$ degree of freedom 


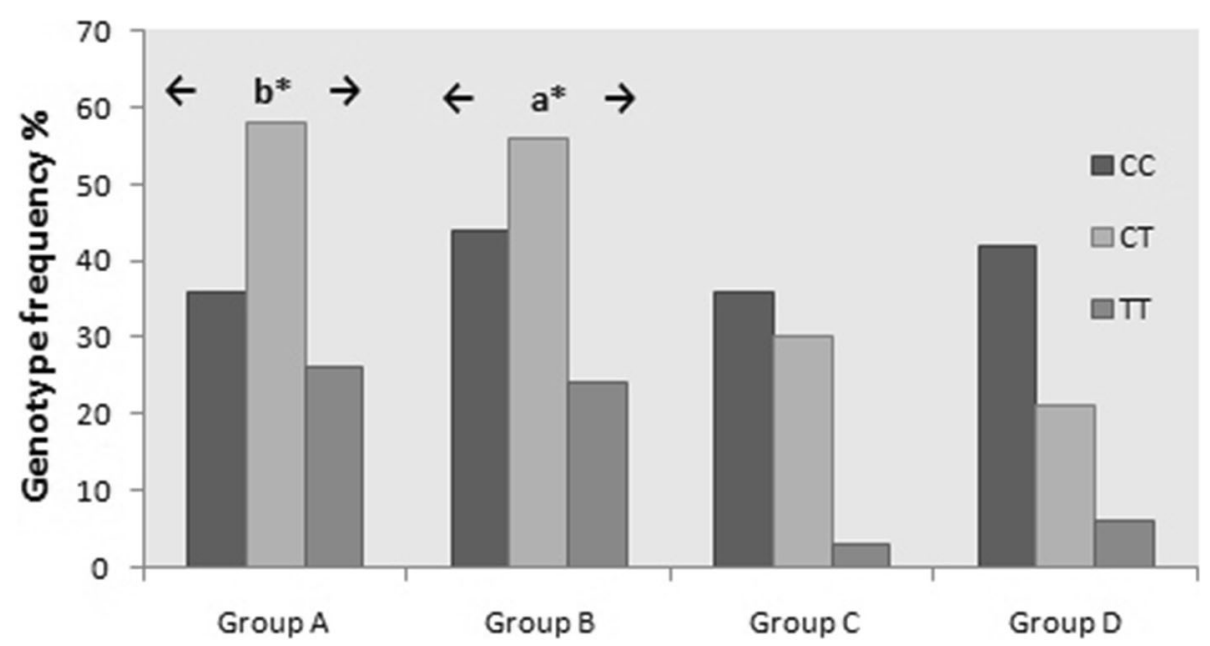

Fig. 2 Genotype frequency for VDR polymorphism at FokI (VDR 2228570 C > T) in all study groups. a, significant difference from group D; b, significant difference from group $C_{;} C$, significant difference from group B; asterisk indicates significant ${ }^{\circ} 0.05$

pressure (SBP) and diastolic blood pressure (DBP) were seen in patients with CT genotype, than those with $\mathrm{CC}$ genotype, while lower HDL-C was seen in patients having TT genotype. In addition, higher FBG, HOMA-IR, cholesterol, triglyceride, and LDL-C were seen in patients with TT genotype, than CC and CT genotype, whereas lower Vit.D levels were seen in patients with TT genotype than CC and CT genotype (Table 6). In CVD patients with T2D, FokI VDR polymorphism showed that TT carriers had higher cholesterol, triglyceride, HDL-C levels than CC and CT genotypes, while TT carriers showed lower Vit.D levels than with CC and CT genotypes. FokI VDR polymorphism in CVD patients with T2D showed no significant association with SBP and DBP (Table 6).

Table 7 presents the distribution of some clinical and biochemical parameters according to the VDR at BsmI (rs1544410 A > G) genotype observed in CVD with and without T2D patients. The results demonstrated that patients with CVD without T2D carrying GG genotype had higher SBP and DBP than patients with AA genotype. Also, the GG genotype was associated with higher FBG, HOMA-IR, cholesterol, triglyceride, and LDL-C levels than AA and AG genotypes in CVD patients without T2D. Vit.D levels were lower in CVD without T2D patients with GG genotype than

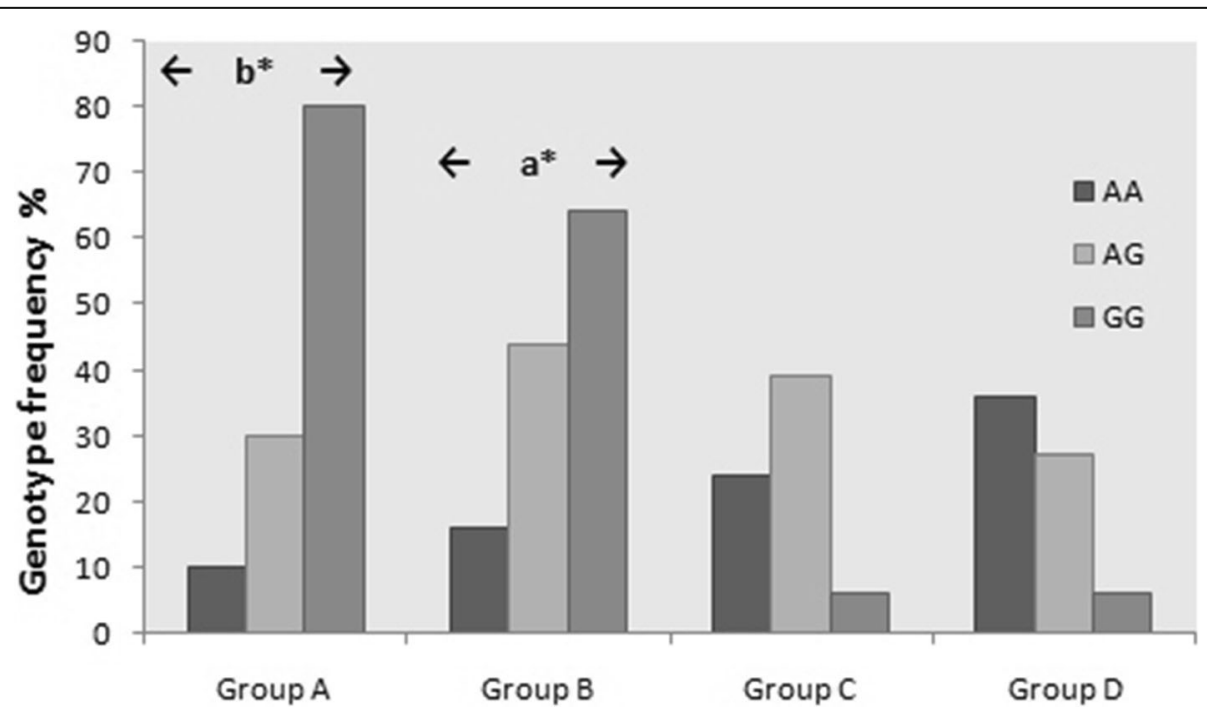

Fig. 3 Genotype frequency for VDR polymorphism at Bsm/ (VDR 1544410 A > G) in all study groups. a, significant difference from group D; b, significant difference from group $C ; C$, significant difference from group $B$; asterisk indicates significant ${ }^{`} 0.05$ 
Table 4 Genotype and allelic frequency for VDR polymorphism at Fokl (rs2228570 C > T)

\begin{tabular}{|c|c|c|c|c|c|}
\hline rs2228570 (Fokl) & $\begin{array}{l}\text { Group A } \\
(n=120)\end{array}$ & $\begin{array}{l}\text { Group B } \\
(n=124)\end{array}$ & $\begin{array}{l}\text { Group C } \\
(n=69)\end{array}$ & $\begin{array}{l}\text { Group D } \\
(n=69)\end{array}$ & $P$ values \\
\hline \multicolumn{6}{|l|}{ Genotype } \\
\hline $\mathrm{CC}$ & $36(30.0 \%)$ & $44(35.5 \%)$ & $36(52.2 \%)$ & $42(60.9 \%)$ & $<0.001$ \\
\hline $\mathrm{CT}$ & $58(48.3 \%)$ & $56(45.2 \%)$ & $30(43.5 \%)$ & $21(30.4 \%)$ & \\
\hline $\mathrm{TT}$ & $26(21.7 \%)$ & $24(19.4 \%)$ & $3(4.3 \%)$ & $6(8.7 \%)$ & \\
\hline $\mathrm{CT}+\mathrm{TT}$ & $84(70 \%)$ & $80(64.6 \%)$ & $33(47.8 \%)$ & $27(39.1 \%)$ & $<0.001$ \\
\hline$P$ value & $\begin{array}{l}0.001^{\mathrm{b} *} \\
0.654^{\mathrm{c}}\end{array}$ & $\begin{array}{l}0.002^{\mathrm{a} *} \\
0.654^{\mathrm{c}}\end{array}$ & $0.218^{a}$ & & \\
\hline Hardly Weinberg equilibrium test $p$ value & 0.77 & 0.41 & 0.28 & 0.17 & \\
\hline \multicolumn{6}{|l|}{ Allele } \\
\hline C & $130(54.2 \%)$ & $144(58.1)$ & $102(73.9 \%)$ & $105(76.1 \%)$ & $<0.001$ \\
\hline $\mathbf{T}$ & $110(45.8 \%)$ & $104(41.9 \%)$ & $36(26.1 \%)$ & $33(23.9 \%)$ & \\
\hline$P$ value & $\begin{array}{l}<0.001^{b_{*}} \\
0.386^{c}\end{array}$ & $<0.001^{\mathrm{a}^{*}}$ & $0.301^{a}$ & & \\
\hline OR $(95 \% \mathrm{Cl})$ & $\begin{array}{l}2.397(1.518-3.786)^{b} \\
1.172(0.819-1.676)^{c}\end{array}$ & $2.298(1.443-3.660)$ & $1.42(0.79-2.54)$ & & \\
\hline
\end{tabular}

Chi-square $(x 2)$ test was used to get the statistical variation. a, significant difference from group $D ; b$, significant difference from group $C$; $c$ significant difference from group $B$

those with AA and AG genotype. Regarding patients with CVD with T2D carrying GG genotype, they presented no difference in SBP and DBP compared to patients with AA genotype, while genotype GG was associated with higher FBG, HOMA-IR, cholesterol, triglyceride, and LDL-C levels than AA and AG genotypes. Vit.D levels were lower in CVD with T2D patients with GG genotype compared to those with AA and AG genotype.

\section{Discussion}

CVD is the leading global cause of death and accounts for more than a third of all deaths worldwide. In Egypt, CVDs represent $40 \%$ of total deaths. CVD is influenced by genetics and environmental risk factors. The most important risk factors of heart disease are unhealthy diets, physical inactivity, high cholesterol levels, hypertension, obesity, smoking, genetics, aging, and diabetes [10]. Vit.D plays a role in a broad range of functions other

Table 5 Genotype and allele frequency for VDR polymorphism at Bsml (rs1544410 A > G)

\begin{tabular}{|c|c|c|c|c|c|}
\hline rs1544410 (Bsml) & $\begin{array}{l}\text { Group A } \\
(n=120)\end{array}$ & $\begin{array}{l}\text { Group B } \\
(n=124)\end{array}$ & $\begin{array}{l}\text { Group C } \\
(n=69)\end{array}$ & $\begin{array}{l}\text { Group D } \\
(n=69)\end{array}$ & $P$ values \\
\hline \multicolumn{6}{|l|}{ Genotype } \\
\hline AA & $10(8.3 \%)$ & $16(12.9 \%)$ & $24(34.8 \%)$ & $36(52.2 \%)$ & $<0.001$ \\
\hline AG & $35(29.0 \%)$ & $44(35.5 \%)$ & $39(56.5 \%)$ & $27(39.1 \%)$ & \\
\hline GG & $75(62.7 \%)$ & $64(51.6 \%)$ & $6(8.7 \%)$ & $6(8.7 \%)$ & \\
\hline AG+GG & $110(91.7 \%)$ & $108(87.1 \%)$ & $45(65.2 \%)$ & $33(47.8 \%)$ & $<0.001$ \\
\hline$P$ value & $\begin{array}{l}<0.001^{\text {b* }} \\
0.057^{c}\end{array}$ & $<0.001^{\mathrm{a}^{*}}$ & $0.101^{\mathrm{a}}$ & & \\
\hline Hardly Weinberg equilibrium test $p$ value & 0.056 & 0.065 & 0.07 & 0.77 & \\
\hline \multicolumn{6}{|l|}{ Allele } \\
\hline A & $55(22.4 \%)$ & $76(31.0 \%)$ & $87(63.8 \%)$ & 99 (73.2\%) & $<0.001$ \\
\hline G & $185(77.6 \%)$ & $172(69.0 \%)$ & $51(36.2 \%)$ & $39(26.8 \%)$ & \\
\hline$P$ value & $\begin{array}{l}<0.0011^{b_{*}} \\
0.013^{c^{*}}\end{array}$ & $<0.001^{\mathrm{a}^{*}}$ & $0.06^{\mathrm{a}}$ & & \\
\hline OR (95\% Cl) & $\begin{array}{l}6.482(4.07-10.32)^{b} \\
1.679(1.11-2.53)^{c}\end{array}$ & $5.745(3.63-9.08)$ & $1.55(0.929-2.58)$ & & \\
\hline
\end{tabular}

Chi-square (x2) test was used to get the statistical variation. a, significant difference from group D; b, significant difference from group $C$; $c$ significant difference from group $B$ 
Table 6 Association between different study parameters and gene polymorphism for VDR at rs2228570 (Fokl) in CVD patients without and with diabetes

\begin{tabular}{|c|c|c|c|c|c|c|}
\hline \multirow{2}{*}{$\begin{array}{l}\text { Groups and genotypes } \\
\text { Variables }\end{array}$} & \multicolumn{6}{|c|}{ CVD without T2D $(n=124)$} \\
\hline & $\begin{array}{l}\mathrm{CC} \\
n=44\end{array}$ & $\begin{array}{l}\mathrm{CT} \\
\mathrm{n}=56\end{array}$ & $\begin{array}{l}\mathrm{TT} \\
\mathrm{n}=24\end{array}$ & $\begin{array}{l}\text { Kruskal- } \\
\text { Wallis } \\
P \text { value }\end{array}$ & $\begin{array}{l}\mathrm{CT}+\mathrm{TT} \\
\mathrm{n}=80\end{array}$ & $\mathrm{CT}+\mathrm{TT} / \mathrm{CC}$ \\
\hline BMI $\left(\mathrm{kg} / \mathrm{m}^{2}\right)$ & $28.8(27.4-33.2)$ & $29.6(28.3-32.8)$ & $32.8(30.9-37.1)$ & $<0.001$ & $31.2(28.5-33.9)$ & $<0.05$ \\
\hline SBP $(\mathrm{mmHg})$ & $130(120-140)$ & $140(130-150)$ & $140(130-140)$ & $<0.001$ & $140(130-150)$ & $<0.001$ \\
\hline DSP (mmHg) & $80(80-90)$ & $85(80-97.5)$ & $80(80-90)$ & $<0.05$ & $80(80-90)$ & $<0.05$ \\
\hline FBG (mg/dl) & $85.7(81.8-90.6)$ & $90.4(83.4-96.6)$ & $110.3(86.5-115.3)$ & $<0.001$ & $93(84.1-104.9)$ & $<0.01$ \\
\hline Insulin ( / ) & $11.5(9.4-15.4)$ & $16.5(9.5-25.1)$ & $10.1(6.1-38.8)$ & 0.11 & $15.8(8.8--26.5)$ & $<0.05$ \\
\hline HOMA-IR & $2.3(1.9-3.2)$ & $3.5(2-5.9)$ & $2.8(1.6-9.4)$ & $<0.05$ & $3.3(2-6.5)$ & $<0.05$ \\
\hline $\mathrm{TC}(\mathrm{mg} / \mathrm{dl})$ & $197.6(185.6-203.3)$ & $206.9(177.3-220.1)$ & $226.6(201.3-254.8)$ & $<0.001$ & $211(181.5-225.3)$ & $<0.01$ \\
\hline TG (mg/dl) & $126.5(97-145)$ & $145.6(123.7-158)$ & $167(146.4-180.2)$ & $<0.001$ & $152.2(130.1-165.6)$ & $<0.01$ \\
\hline HDL-C (mg/dl) & $48.75(40-56.4)$ & $42.1(34.2-45.6)$ & $36(24-54.8)$ & $<0.05$ & $40.7(32.4-48.2)$ & $<0.01$ \\
\hline LDL-C (mg/dL) & $124.2(113.2-144.3)$ & $136.1(115.8-144.3)$ & $141.4(116.5-191.3)$ & 0.054 & 137.5 (115.9-150.5) & 0.57 \\
\hline Vit.D (ng/ml) & $20.6(18.1-25.5)$ & $17(13.7-21.1)$ & $8.8(7.2-10.3)$ & $<0.001$ & $16.3(10-19.2)$ & $<0.001$ \\
\hline \multirow{2}{*}{$\begin{array}{l}\text { Groups and genotypes } \\
\text { Variables }\end{array}$} & \multicolumn{6}{|c|}{ CVD with T2D $(n=120)$} \\
\hline & $\begin{array}{l}\mathrm{CC} \\
n=36\end{array}$ & $\begin{array}{l}\mathrm{CT} \\
\mathrm{n}=58\end{array}$ & $\prod_{n=26}^{T T}$ & $\begin{array}{l}\text { Kruskal-Wallis } \\
P \text { value }\end{array}$ & $\begin{array}{l}\mathrm{CT}+\mathrm{TT} \\
\mathrm{n}=84\end{array}$ & $\mathrm{CT}+\mathrm{TT} / \mathrm{CC}$ \\
\hline BMI $\left(\mathrm{kg} / \mathrm{m}^{2}\right)$ & $28.5(25-30.4)$ & $30.8(27.6-33.2)$ & $31(28.8-33.4)$ & $<0.01$ & $30.8(28.1-33.2)$ & $<0.01$ \\
\hline SBP $(\mathrm{mmHg})$ & $140(130-140)$ & $130(117.5-150)$ & $140(130-150)$ & 0.62 & 140 (120-150) & 0.67 \\
\hline $\mathrm{DSP}(\mathrm{mmHg})$ & $80(80-90)$ & $90(77.5-100)$ & $90(80-90)$ & 0.7 & $90(80-90)$ & 0.5 \\
\hline FBG (mg/dl) & $154.2(137.4-180)$ & 196.7 (181.4-241.8) & $210.6(168.5-226.9)$ & $<0.001$ & 196.7 (178.4-240.7) & $<0.001$ \\
\hline Insulin ( / $)$ & $23.3(14.6-28.5)$ & $28.5(19.5-42.6)$ & $30.4(16-63.4)$ & 0.07 & $29.4(18.6-53.2)$ & $<0.05$ \\
\hline HOMA-IR & $9.7(5.7-13.1)$ & $12.8(9.42-26)$ & $14.2(7.4-31.1)$ & $<0.01$ & $13.2(8.1-29.4)$ & $<0.01$ \\
\hline $\mathrm{TC}(\mathrm{mg} / \mathrm{dl})$ & $191.6(184.3-205.9)$ & $200.5(197.2-216.2)$ & $259.5(202.5-271.8)$ & $<0.001$ & $204.9(189.2-250.4)$ & $<0.001$ \\
\hline TG (mg/dl) & $134.9(100.7-156)$ & $140.6(128.5-167.6)$ & $176.5(155.6-198.7)$ & $<0.001$ & $151.7(136.8-167.5)$ & $<0.01$ \\
\hline HDL-C (mg/dl) & $41.6(38.2-55.2)$ & $36.8(30.3-40.6)$ & $30.5(24.4-37.2)$ & $<0.001$ & $35.5(28.6-40.5)$ & $<0.01$ \\
\hline LDL-C (mg/dL) & $125.7(110.3-142.3)$ & $136.8(126.5-152.6)$ & $179.8(141-204.4)$ & $<0.001$ & $140.9(129.1-179.7)$ & $<0.001$ \\
\hline Vit.D (ng/ml) & $16.9(15.2-18.8)$ & $12.8(10.5-14.5)$ & $6.5(4.6-9.1)$ & $<0.001$ & $12.5(8-14.2)$ & $<0.001$ \\
\hline
\end{tabular}

Data were expressed as median (interquartile range, IQR: 25th-75th quartile) and compared using the non-parametric Kruskal-Wallis test

than bone health, including cardiovascular health [31]. Several studies have reported a conflicting association between Vit.D deficiency, VDR polymorphism, and the risk of CVD [22, 32]. Recently, linked analysis of VDR polymorphisms and Vit.D levels represented the approved method of investigation of many diseases [33]. The present study examined the correlation of Vit.D level in CVD patients with and without T2D in the Egyptian population. We found decreased Vit.D level in CVD patients (both with and without T2D) than diabetic patients and healthy controls. In addition, diabetic CVD patients had the lowest levels of Vit.D compared to those without diabetes. Our results are in line with Ma et al. [33], who observed a significantly lower level of serum Vit.D in T2D subjects with and without CVD compared to healthy subjects. Also, these data agreed with the studies of Fox et al. [34] and Tarighi et al. [35] that revealed a correlation between low Vit.D and the incidence of CVD in patients with diabetes. The Vit.D relationship with the cardiovascular system has been illustrated by epidemiological studies elucidating the association of decreased Vit.D level with CVD. Nargesi et al. [27] and Kavarie et al. [36] studies demonstrated that the low levels of Vit.D shown in patients with stroke, myocardial infarction, and heart failure as Vit.D lower renin gene expression, modulates the growth and proliferation of both cardiomyocytes and vascular smooth muscle cells. The absence of VDR activation results in the upregulation of the rennin angiotensin system, hypertension, endothelial dysfunction, and left ventricular hypertrophy [37]. However, the role of Vit.D binding protein in the pathogenesis of CVD remains conflicting and not clear. Similar results have been achieved in more cohort studies documenting an 
Table 7 Association between different study parameters and different VDR at rs1544410 A > G (Bsml) genotypes in CVD patients without and with diabetes

\begin{tabular}{|c|c|c|c|c|c|c|}
\hline \multirow{2}{*}{$\begin{array}{l}\text { Groups and } \\
\text { genotypes } \\
\text { Variables }\end{array}$} & \multicolumn{6}{|c|}{ CVD without T2D $(n=124)$} \\
\hline & $\begin{array}{l}A A \\
n=16\end{array}$ & $\begin{array}{l}\mathrm{AG} \\
\mathrm{n}=44\end{array}$ & $\begin{array}{l}\mathrm{GG} \\
\mathrm{n}=64\end{array}$ & $\begin{array}{l}\text { Kruskal-Wallis } \\
P \text { value }\end{array}$ & $\begin{array}{l}A G+G G \\
n=108\end{array}$ & $A G+G G / A A$ \\
\hline BMI $\left(\mathrm{kg} / \mathrm{m}^{2}\right)$ & $28.2(26.9-30)$ & $29.2(27.1-30.7)$ & $32.5(29.3-34.5)$ & ${ }^{\circ} 0.001$ & $31.2(28.5-33.9)$ & ${ }^{\circ} 0.01$ \\
\hline $\mathrm{SBP}(\mathrm{mmHg})$ & $130(112.5-130)$ & $130(120-140)$ & 140(130-150) & ${ }^{<} 0.01$ & $140(130-150)$ & $<0.05$ \\
\hline $\mathrm{DSP}(\mathrm{mmHg})$ & $70(70-87.5)$ & $80(80-90)$ & $80(80-90)$ & ${ }^{<} 0.01$ & $80(80-90)$ & $<0.01$ \\
\hline FBG (mg/dl) & $88.8(82-100.6)$ & $85.3(79.9-88.5)$ & $95.2(88.8-106.8)$ & $<0.001$ & $93(84.1-104.9)$ & 0.85 \\
\hline Insulin ( /I) & $8.8(7.4-17)$ & $12.2(10-15.4)$ & $18.1(9.4-28)$ & ${ }^{<} 0.05$ & $15.8(8.8-26.5)$ & $<0.05$ \\
\hline HOMA-IR & $1.9(1.5-3.5)$ & $2.4(2-3.3)$ & $3.9(2-6.8)$ & ${ }^{<} 0.05$ & $3.3(2-6.5)$ & $<0.05$ \\
\hline $\mathrm{TC}(\mathrm{mg} / \mathrm{dl})$ & $185.6(177.3-195.3)$ & $198.8(189.4-205.9)$ & $219.6(198.8-225.3)$ & $<0.001$ & $211(181.5-225.3)$ & ${ }^{<} 0.01$ \\
\hline $\mathrm{TG}(\mathrm{mg} / \mathrm{dl})$ & $101.4(83.1-159.4)$ & $124.8(97-140.6)$ & $157.5(140.9-169.2)$ & $<0.001$ & $152.2(130.1-165.6)$ & $<0.05$ \\
\hline $\mathrm{HDL}-\mathrm{C}(\mathrm{mg} / \mathrm{dl})$ & $57.7(50.3-63.3)$ & $46.4(34.8-55.2)$ & $38.2(31.4-44.7)$ & $<0.001$ & $40.7(32.4-48.2)$ & $<0.001$ \\
\hline $\mathrm{LDL}-\mathrm{C}(\mathrm{mg} / \mathrm{dL})$ & $108.4(96.9-124.3)$ & $125.3(116.9-140.4)$ & $140.3(116.5-156.9)$ & $<0.001$ & $137.5(115.9-150.5)$ & $<0.001$ \\
\hline Vit.D (ng/ml) & $25.7(21.4-27.4)$ & $20.1(17.8-22.4)$ & $12.9(9.7-16.9)$ & $<0.001$ & $16.3(10-19.2)$ & $<0.001$ \\
\hline \multirow{2}{*}{$\begin{array}{r}\text { Groups andgenotypes } \\
\text { Variables }\end{array}$} & \multicolumn{6}{|c|}{ CVD with T2D $(n=120)$} \\
\hline & $\begin{array}{l}A A \\
n=10\end{array}$ & $\begin{array}{l}A G \\
n=35\end{array}$ & $\begin{array}{l}\mathrm{GG} \\
\mathrm{n}=75\end{array}$ & $\begin{array}{l}\text { Kruskal-Wallis } \\
P \text { value }\end{array}$ & $\begin{array}{l}A G+G G \\
n=110\end{array}$ & $A G+G G / A A$ \\
\hline BMI $\left(\mathrm{kg} / \mathrm{m}^{2}\right)$ & $26.1(22.9-31.5)$ & $29.3(26.2-30.8)$ & $30.4(27.7-33.2)$ & 0.07 & $29.4(27.6-33.2)$ & $<0.05$ \\
\hline $\mathrm{SBP}(\mathrm{mmHg})$ & 130 (120-145) & $140(120-150)$ & $140(130-150)$ & 0.062 & $140(130-150)$ & 0.45 \\
\hline $\mathrm{DSP}(\mathrm{mmHg})$ & $80(75-90)$ & $90(80-100)$ & $90(80-90)$ & 0.12 & $90(80-90)$ & 0.15 \\
\hline FBG (mg/dl) & $150(128.9-209.1)$ & $159.5(139.1-182.4)$ & $196.3(178.1-245.5)$ & $<0.001$ & $189.4(159.5-239)$ & $<0.05$ \\
\hline Insulin ( /I) & $14.2(8.4-31.9)$ & $21(13.4-31.3)$ & $28.5(19.6-54.4)$ & ${ }^{<} 0.01$ & $27.4(17.4-47.8)$ & $<0.05$ \\
\hline HOMA-IR & $7.6(3.6-10.4)$ & $7.4(5.7-13.1)$ & $13.6(10-34.1)$ & $<0.001$ & $12.6(7.1-25.1)$ & $<0.01$ \\
\hline $\mathrm{TC}(\mathrm{mg} / \mathrm{dl})$ & $202.1(171.1-204)$ & $195.2(184.8-205.9)$ & $207.5(198.2-254.4)$ & $<0.001$ & $200.5(192.8-223.4)$ & 0.1 \\
\hline $\mathrm{TG}(\mathrm{mg} / \mathrm{dl})$ & $137.5(96.9-153.2)$ & $156(130.6-168.9)$ & $147.8(124.8-178.7)$ & 0.169 & $148.6(130-175.3)$ & 0.07 \\
\hline HDL-C (mg/dl) & $53(44.2-58.1)$ & $42.3(36.8-55.2)$ & $33.2(27.1-38.8)$ & $<0.001$ & $36.7(28.6-41.2)$ & $<0.001$ \\
\hline LDL-C (mg/dL) & $126(94-128.3)$ & $124.1(101-142.3)$ & $144.2(132-180)$ & $<0.001$ & $139.5(125.9-166.2)$ & ${ }^{<} 0.05$ \\
\hline Vit.D (ng/ml) & $18.1(14.4-22.2)$ & $15.2(13.4-17.8)$ & $12.3(7.8-14.5)$ & $<0.001$ & $12.8(10-15.8)$ & $<0.01$ \\
\hline
\end{tabular}

Data were expressed as median (interquartile range, IQR: 25th-75th quartile) and compared using the non-parametric Kruskal-Wallis test

association between decreased Vit.D levels and angiographic definition of coronary artery disease (CAD) and platelet reactivity [32, 37]. In agreement with Judd et al. [38], our logistic regression analysis showed that Vit.D is a CVD risk factor. Also, in partial agreement with Nakhl et al. [20] and Orozco-Beltran et al. [39], BMI, FBG, insulin, and HOMA-IR are independent risk factors for CVD. However, the lipid profile parameters did not show any significance. The results of the present study could be explained by the fact that each individual parameter of the lipid profile might not be a CVD risk factor; however, the ratios and combinatorial changes between these parameters may be considered as risk factors.

Several polymorphisms have been identified in the VDR. Among them, the most controversial SNPs are the rs2228570 $\mathrm{C}>\mathrm{T}$ and rs1544410 A > G, which are identified by FokI and BsmI restriction enzymes. Research conducted on several populations to detect association of VDR polymorphisms with vulnerability to CVD provided conflicting results [21, 22]. Furthermore, FokI and $B s m I$ variants and minor alleles were implicated in CVD predisposition [16, 19]. With regard to the Egyptian population, we found that the genotype CT within VDR rs2228570 (FokI) occurred more frequently in CVD patients with and without T2D vs. controls. In addition, the allele $\mathrm{T}$ is significantly more frequent in CVD patients with and without T2D and correlated with increased CVD risk. However, the AG genotype within VDR rs1544410 (BsmI) occurred less frequently in CVD patients with and without T2D vs. controls, and the GG genotype was found more frequently in CVD with and without T2D patients. Assumably, the A allele may have a protective effect regarding CVD risk. On the other hand, the $G$ allele may express a high risk. Relating to our findings, these polymorphisms may indicate a new 
cardiac risk factor. Our study corroborated with the earlier observations that in T2D patients with CAD phenotype, the variants of FokI were significantly higher than in those without CAD, suggesting the implication of VDR polymorphisms on CAD susceptibility in patients with T2D [33]. Our results are also in accordance with Nakhl et al. [21], who reported that FokI polymorphism was frequent in Mediterranean subjects with cardiovascular risk factors. FokI SNP is thought to be an independent indicator of the VDR gene, because it is not in linkage disequilibrium with other VDR SNPs and it has a relationship with the functionality of VDR protein [16]. FokI SNP extends the length of the receptor protein and modifies the amino acid sequence of VDR protein, while $B s m I$ is intronic and cannot modify the amino acid sequence of VDR protein. In contrast, a Chinese study by Pan et al. [22] showed no significant evidence of association of both genotype and allele frequencies of FokI and BsmI polymorphisms with CVD. Similar in nature, the study in the Caucasian population revealed no significant difference in biochemical features of CVD between FokI genotypes [40]. In partial agreement, Abu el Maaty et al. [41] stated that the FokI polymorphism of VDR is a potential genetic marker for CAD. With regard to BsmI polymorphism, a cohort study from a multinational perspective found that the minor allele of BsmI correlated with increased CAD risk in T2D patients [19]. In contrast to our results, Ortlepp et al. [18] observed that BsmI polymorphism was associated with the severity of CAD.

Relating the clinical parameters and VDR polymorphisms, it was observed that heterozygotes CT/AG or homozygous TT/GG mutants of FokI and BsmI polymorphism, respectively, had lower levels of Vit.D and were significantly more frequent in diabetic CVD patients than those without diabetes. These results indicate the pivotal function of VDR variants and Vit.D in the progression of CVD in patients with T2D. However, the definite mechanism of CVD predisposition association is not well-known. Reasonably, decreased levels of Vit.D and genetic variants of VDR alter Vit.D expression and can promote cardiac abnormalities. Lipid profile parameters showed significant association with FokI and BsmI polymorphism in CVD patients with and without T2D. Our study showed increased levels of TG, LDL-C, TC, and decreased level of HDL-C in TT and CT, GG, and AG genotype carriers. This result is in accordance with the study of Schuch et al. [42] that stated that FokI VDR gene polymorphism was linked with decreased Vit.D levels, and may be related to decreased HDL-C level and increased levels of TC, TG, and LDL-C. Also, Prabhakar et al. [43] reported that the FokI variant may be linked to cholesterol levels in subjects with ischemic stroke. There are two possible mechanisms by which Vit.D can affect lipid profiles. First, Vit.D increases intestinal calcium absorption and this may reduce triglyceride levels by lowering hepatic triglyceride formation and secretion. Second, Vit.D has a suppressive effect on parathyroid hormone $(\mathrm{PTH})$ secretion, which can reduce lipolysis [44]. In CVD patients with T2D, we noticed significant associations between FokI and BsmI polymorphisms and each of FBG, insulin levels, and HOMA-IR and Vit.D levels. Vit.D affects glucose homeostasis and insulin sensitivity via different mechanisms, including modification and alteration of insulin secretion, calcium metabolism, cytokine expression, and adipocyte function. Accordingly, Vit.D enhances and improves $\beta$-cell function and insulin sensitivity [45].

\section{Limitations of the study}

Although we tried to present a complete image of the CVD risk factors especially in CAD disease and VDR variants, only two VDR gene SNPs were examined and relatively small cohorts of subjects were compared.

\section{Conclusion}

The present study concludes that the level of Vit.D and the distribution of VDR polymorphisms are associated with risk of CVD in Egyptian patients with or without diabetes. These results suggest VDR polymorphisms as potential diagnostic biomarkers for CVD susceptibility. We are recommending further studies addressing the rest of VDR SNPs in larger samples of Egyptian subjects, which will provide more targets to avoid the risk of CVDs.

\section{Abbreviations \\ CVD: Cardiovascular disease; CAD: Coronary artery disease; Vit.D: Vitamin D; VDR: Vitamin D receptor; BMI: Body mass index; FBG: Fasting blood glucose; SNP: Single nucleotide polymorphism; UTR: Untranslated region; T2D: Type 2 diabetes mellitus; DBP: Diastolic blood pressure; SBP: Systolic blood pressure; ECG: Leads resting electrocardiogram; WHO: World Health Organization; PTH: Parathyroid hormone; RAAS: Renin-angiotensin-aldosterone System; TC: Total cholesterol; TG: Triglyceride; HD: High-density lipoprotein; LDL: Low- density lipoprotein; ELISA: Enzyme-linked immunosorbent assay; HOMA- IR: Homeostatic Model Assessment of Insulin Resistance; RFLP: Restriction fragment length polymorphism; DW: Distilled water; OR: Odds ratio; $\mathrm{Cl}$ : Confidence interval}

\section{Supplementary Information}

The online version contains supplementary material available at https:/doi. org/10.1186/s43042-021-00174-9.

Additional file 1: Supplementary figure 1. Agarose gel electrophoresis of VDR polymorphism. Supplementary figure 2. Allele frequency for VDR polymorphism at Fokl (VDR 2228570 C > T) in all study groups. Supplementary figure 3. Allele frequency for VDR polymorphism at Bsm/ (VDR $1544410 \mathrm{~A}>\mathrm{G}$ ) in all study groups.

Acknowledgements

We thank all patients for their participation, and Dr. Ibrahim, Diagnostic Outpatient Clinic, Department of Cardiology in Al-Azhar University for his assistance. 


\section{Authors' contributions}

Conceived and designed the study: A.S., Y.M.S, and N.S. Diagnosis and selection all participants in the study: I.Y. Contributed reagents/materials/analysis tools: S.M.E, N.S., and Y.M.S. Finally, S.M.E., N.S., and R.H.M. contributed to the analysis of the results and to the writing of the manuscript. All authors provided critical feedback and helped shape the research and analysis. The authors read and approved the final manuscript.

\section{Funding}

This work was supported by the National Research Centre, Dokki, Giza, Egypt $[11 / 2 / 4]$. The sponsor had role in the study design, in the collection, and analysis.

\section{Availability of data and materials}

The data that support the findings of this study are available from the corresponding author upon reasonable request.

\section{Declarations}

\section{Ethics approval and consent to participate}

Written informed consent was obtained from each participant prior to the study. The study protocol was approved by the Human Ethical Committee of the National Research Centre (Code No. 16049).

\section{Consent for publication}

Not applicable

\section{Competing interests}

The authors declare that they have no competing interests.

\section{Author details}

'Department of Biochemistry, National Research Centre, Dokki, Giza, Egypt. ${ }^{2}$ Department of Biochemistry, Faculty of Science, Ain Shams University, Cairo, Egypt. ${ }^{3}$ Department of Cardiology, Faculty of Medicine, Al-Azhar University, Cairo, Egypt.

\section{Received: 10 January 2021 Accepted: 4 May 2021}

\section{Published online: 10 June 2021}

\section{References}

1. Mladěnka P, Applová L, Patočka J, Costa VM, Remiao F, Pourová J, Mladěnka A, Karlí̌cková J, Jahodáŕ L, Vopršalová M, Varner K, Štěrba M, TOX-OER and CARDIOTOX Hradec Králové Researchers and Collaborators (2018) Comprehensive review of cardiovascular toxicity of drugs and related agents. Med Res Rev 38(4):1332-1403. https://doi.org/10.1002/med.21476

2. Teo K, Dokainish $\mathrm{H}$ (2017) The emerging epidemic of cardiovascular risk factors and atherosclerotic disease in developing countries. Can J Cardiol 33(3):358-365. https://doi.org/10.1016/i.cjca.2016.12.014

3. World Health Organization. WHO (2017) Cardiovascular diseases (CVDs). Available from: http://www.who.int/cardiovascular_diseases/en/.

4. World Health Organization (2018) Egypt: World Health Organization-noncommunicable diseases (NCD) country profiles, Availablefrom: https://www.who.int/nmh/countries/2018/egy_en.pdf?ua=1.

5. Bhatnagar A (2017) Environmental determinants of cardiovascular disease. Circ Res 121(2):162-180. https://doi.org/10.1161/CIRCRESAHA.117.306458

6. Laakso M, Kuusisto J (2014) Insulin resistance and hyperglycaemia in cardiovascular disease development. Nature Reviews Endocrinology 10(5): 293-302. https://doi.org/10.1038/nrendo.2014.29

7. Fiatal S, Ádány R (2018) Application of single-nucleotide polymorphismrelated risk estimates in identification of increased genetic susceptibility to cardiovascular diseases: a literature review. Front Public Health 358(5). https://doi.org/10.3389/fpubh.2017.00358

8. Skaaby T, Thuesen BH, Linneberg A (2017) Vitamin D, cardiovascular disease and risk factors. Dv Exp Med Biol 996:221-230. https://doi.org/10.1007/ 978-3-319-56017-5_18

9. Wimalawansa S (2018) Vitamin D and cardiovascular diseases: causality. J Steroid BiochemMolBiol 175:29-43. https://doi.org/10.1016/j.jsbmb.2016.12.016

10. Lv L, Tan X, Peng X, Bai R, Xiao Q, Zou T, Tan J, Zhang H, Wang C (2020) The relationships of vitamin $D$, vitamin $D$ receptor gene polymorphisms, and vitamin D supplementation with Parkinson's disease. TransINeurodegener 9(34):34. https://doi.org/10.1186/s40035-020-00213-2
11. Szymczak-Pajor I, Śliwińska A (2019) Analysis of association between vitamin D deficiency and insulin resistance. Nutrients 11(4):794. https://doi.org/10.33 90/nu11040794

12. VimaleswaranKS PC, Hyppönen E (2014) Interaction between vitamin D receptor gene polymorphisms and 25-hydroxyvitamin D concentrations on metabolic and cardiovascular disease outcomes. Diabetes Metab 40(5):386395. https://doi.org/10.1016/j.diabet.2014.01.003

13. Jain R, Von Hurst PR, Stonehouse W et al (2012) Association of vitamin D receptor gene polymorphisms with insulin resistance and response to vitamin D. Metabolism 61(3):293-301. https://doi.org/10.1016/j.metabol.2011.06.018

14. Zaki M, Kamal S, Basha WA, Youness E, Ezzat W, el-Bassyouni H, Amr K (2017) Association of vitamin D receptor gene polymorphism (VDR) with vitamin D deficiency, metabolic and inflammatory markers in Egyptian obese women. Genes \& Diseases 4(3):176-182. https://doi.org/10.1016/j. gendis.2017.07.002

15. Shen F, Guo C, Wang Y, Yu F, Zhang D, Liu X, Ba Y, Wang C, Li W, Li X (2020) Lowserum 25-hydroxyvitamin D levels may increase the detrimental effect of VDR variants on the risk of essential hypertension. Eur J ClinNutr 74:1091-1099. https://doi.org/10.1038/s41430-019-0543-5

16. Maia J, Da Silva AS, Do Carmo RF et al (2016) The association between vitamin D receptor gene polymorphisms (Taql and Fokl), Type 2 diabetes, and micro-/macrovascular complications in postmenopausal women. Appl Clin Genet 9:131-136. https://doi.org/10.2147/TACG.S101410

17. Hassan I, Bhat YJ, Majid S et al (2019) Association of vitamin D receptor gene polymorphisms and serum 25-hydroxy vitamin D levels in vitiligo - a case-control study. Indian Dermatol Online J10 2:131-138. https://doi.org/1 0.4103/idoj.IDOJ_97_18

18. Ortlepp JR, Lauscher J, Hoffmann R, Hanrath P, Joost HG (2001) The VDR gene variant is associated with the prevalence of Type 2 diabetes mellitus and coronary artery disease. Diabet Med 18(10):842-845. https://doi.org/10.1 046/j.1464-5491.2001.00585.x

19. Ferrarezi DA, Bellili-Muñoz N, Dubois-Laforgue D et al (2013) Allelic variations of the vitamin $D$ receptor (VDR) gene are associated with increased risk of coronary artery disease in type 2 diabetics: the DIABHYCAR prospective study. Diabetes Metab 39(3):263-270

20. JiaJ TY, Shen C et al (2018) Vitamin D receptor polymorphism rs 2228570 is significantly associated with risk of dyslipidemia and serum LDL levels in Chinese Han population. Lipids Health Dis 17(193) https://doi.org/10.1186/ s12944-018-0819-0:193

21. Nakhl S, Sleilaty G, Chouery E et al (2019) Fokl vitamin D receptor gene polymorphism and serum 25-hydroxyvitamin D in patients with cardiovascular risk. Arch Med SciAtheroscler Dis 4:e298-e303

22. Pan XM, Li DR, Yang L, Wang EY, Chen TY, Liu YJ, Liu M, Liao ZG (2009) No association between vitamin $D$ receptor polymorphisms and coronary artery disease in a Chinese population. DNA Cell Biol 28(10):521-525. https://doi. org/10.1089/dna.2009.0908

23. Abu el Maaty MA, Hassanein SI, Gad MZ (2016) Genetic variation in vitamin D receptor gene (Fok1:rs2228570) is associated with risk of coronary artery disease. Biomarkers 21(1):68-72

24. World Health Organization (2007) Prevention of cardiovascular disease: guidelines for assessment and management of cardiovascular risk. World Health Organization https://apps.who.int/iris/handle/10665/43685

25. American Diabetes Association (2014) Standards of medical care in diabetes- 2014. Diabetes care 37(Suppl 1):S14-S80. https://doi.org/10.2337/ dc14-5014

26. Friedewald WT, Levy Rl, Fredrickson DS (1972) Estimation of the concentration of low-density lipoprotein cholesterol in plasma without use of the preparative ultracentrifuge. Clin Chem 18(6):499-502. https://doi. org/10.1093/clinchem/18.6.499

27. Nargesi AA, Heidari B, Esteghamati S, Hafezi-Nejad N, Sheikhbahaei S, Pajouhi A, Nakhjavani M, Esteghamati A (2016) Contribution of vitamin D deficiency to the risk of coronary heart disease in subjects with essential hypertension. Atherosclerosis 244:165-171. https://doi.org/10.1016/j.a therosclerosis.2015.11.020

28. Chen XE, Chen P, Chen SS, Lu J, Ma T, Shi G, Zhou Y, Li J, Sheng L (2017) A population association study of vitamin $\mathrm{D}$ receptor gene polymorphisms and haplotypes with the risk of systemic lupus erythematosus in a Chinese population. Immunol Res 65(3):750-756. https://doi.org/10.1007/s12026-0178914-2

29. Cohen J (1988) Statistical power analysis for the behavioral sciences. Lawrence Erlbaum Associates, Hillsdale, New Jersey 
30. Dawson B, Trapp RG (2001) Basic \& clinical biostatistics. Lange Medical Book/ McGraw-Hill, Medical Publishing Division 3rd ed 7-9:161-218.

31. Lee JH, O'Keefe JH, Bell D et al (2008) Vitamin D deficiency an important, common, and easily treatable cardiovascular risk factor? J Am Coll Cardiol 52(24):1949-1956. https://doi.org/10.1016/j.jacc.2008.08.050

32. Verdoia M, Pergolini P, Rolla R, Sartori C, Nardin M, Schaffer A, Barbieri L, Daffara V, Marino P, Bellomo G, Suryapranata H, De Luca G (2016) Vitamin D levels and high-residual platelet reactivity in patients receiving dual antiplatelet therapy with clopidogrel or ticagrelor. Platelets 27(6):576-582

33. Ma L, Wang S, Chen H, Cui L, Liu X, Yang H, Li G, Liu S, Qi T, Tian H (2020) Diminished $25-\mathrm{OH}$ vitamin D3 levels and vitamin $\mathrm{D}$ receptor variants are associated with susceptibility to type 2 diabetes with coronary artery diseases. J Clin Lab Anal 34(4):e23137. https://doi.org/10.1002/jcla.23137

34. Fox H, Seeger FH, Quernheim QF et al (2020) Vitamin D gene polymorphisms and risk of acute cardiovascular events, Clinical Epidemiology and Global Health. 8(4):1371-1376 https://doi.org/10.1016/j. cegh.2020.05.015

35. Tarighi S, Najafi M, Hossein-Nezhad A, Ghaedi H, Meshkani R, Moradi N, Fadaei R, Kazerouni F, Shanaki M (2017) Association between two common polymorphisms of vitamin $\mathrm{D}$ binding protein and the risk of coronary artery disease: a case-control study. J Med Biochem 36(4):349-357. https://doi. org/10.1515/jomb-2017-0015

36. Kavarić S, Vuksanović M, Bozović D et al (2013) Body weight and waist circumference as predictors of vitamin D deficiency in patients with type 2 diabetes and cardiovascular disease. Vojnosanit Pregl 70(2):163-169. https:// doi.org/10.2298/NSP110713035K

37. Pilz S, Tomaschitz A, März W, Drechsler C, Ritz E, Zittermann A, Cavalier E, Pieber TR, Lappe JM, Grant WB, Holick MF, Dekker JM (2011) Vitamin D, cardiovascular disease and mortality. ClinEndocrinol (Oxf) 75(5):575-584. https://doi.org/10.1111/j.1365-2265.2011.04147.x

38. Judd SE, Tangpricha V (2009) Vitamin D deficiency and risk for cardiovascular disease. The American journal of the medical sciences 338(1): 40-44 https://doi.org/10.1097/MAJ.0b013e3181aaee91

39. Orozco-Beltran D, Gil-Guillen VF, Redon J, Martin-Moreno JM, PallaresCarratala V, Navarro-Perez J, Valls-Roca F, Sanchis-Domenech C, FernandezGimenez A, Perez-Navarro A, Bertomeu-Martinez V, Bertomeu-Gonzalez V, Cordero A, Pascual de la Torre M, Trillo JL, Carratala-Munuera C, PitaFernandez S, Uso R, Durazo-Arvizu R, Cooper R, Sanz G, Castellano JM, Ascaso JF, Carmena R, Tellez-Plaza M, on behalf of ESCARVAL Study Group (2017) Lipid profile, cardiovascular disease and mortality in a Mediterranean high-risk population: the ESCARVAL-RISK study. PLoSOne 12(10):e0186196. https://doi.org/10.1371/journal.pone.0186196

40. Laczmanski L, Milewicz A, Lwow F et al (2013) Vitamin D receptor gene polymorphism and cardiovascular risk variables in elderly Polish subjects. GynecolEndocrinol 29(3):268-272

41. Abu el Maaty MA, Hassanein SI, Sleem HM, Gad MZ (2015) Vitamin D receptor gene polymorphisms (Taql and Apal) in relation to $25-$ hydroxyvitamin $\mathrm{D}$ levels and coronary artery disease incidence. Journal of Receptors and Signal Transduction 35(5):391-395. https://doi.org/10.3109/1 0799893.2014.959593

42. Schuch NJ, Garcia VC, Sandra RG et al (2013) Relationship between vitamin $D$ receptor gene polymorphisms and the components of metabolic syndrome. Nutr J 12(96). https://doi.org/10.1186/1475-2891-12-96

43. Prabhakar P, Majumdar V, Kulkarni GB, Christopher R (2015) Genetic variants of vitamin D receptor and susceptibility to ischemic stroke. BiochemBiophys Res Commun 456(2):631-636. https://doi.org/10.1016/j.bbrc.2014.12.007

44. Zittermann A, Gummert JF, Börgermann J (2011) The role of vitamin D in dyslipidemia and cardiovascular disease. Current Pharmaceutical Design 17(9):933-942. https://doi.org/10.2174/138161211795428786

45. Mridula M, Ritu S (2015) Current understanding of role of vitamin D in type 2 DM. Int J Recent Sci Res 6(2):2602-2607

\section{Publisher's Note}

Springer Nature remains neutral with regard to jurisdictional claims in published maps and institutional affiliations.

\section{Submit your manuscript to a SpringerOpen ${ }^{\circ}$ journal and benefit from:}

- Convenient online submission

- Rigorous peer review

- Open access: articles freely available online

- High visibility within the field

- Retaining the copyright to your article

Submit your next manuscript at $\boldsymbol{\nabla}$ springeropen.com 